\title{
The best of both world, how a "Christmas tree" TEM can please biologist and material scientists.
}

\author{
Eric Hanssen ${ }^{1}$, Mat P Watts ${ }^{2}$, Edward R Smith ${ }^{3}$, Prajakta Gosavi ${ }^{4}$, Paul A Gleeson ${ }^{4}$ and John W Moreau ${ }^{2}$ \\ 1. Advance Microscopy Facility, Bio21 Institute, The University of Melbourne, Melbourne, Australia \\ 2. School of Earth Science, The University of Melbourne, Melbourne, Australia \\ 3. Department of Nephrology, The Royal Melbourne Hospital, Melbourne, Australia \\ 4. Department of Biochemistry and Molecular Biology, The University of Melbourne, Melbourne, \\ Australia
}

Historically the "soft and squishy" (eg. Life science) and the "hard and dry" (eg. Material science) scientific worlds have not mixed really well in the electron microscopy/microanalysis field with poor overlap of capabilities and knowledge within service centers usually dedicated to one or the other. But more and more often the need to save space, save funds and staff sees the two worlds collide. Usually this result in a lot of compromise on the equipment side but can also benefit both parties.

We will take the example of a FEI Tecnai F30 that was originally set up for cellular biology as a cryoTEM, twin pole pieces, cryo box and tomography capability. Recently it has been upgraded with STEM and STEM-EELS. The cryo capability is mainly used by the biologist and the STEM and/or EELS are essentially used in material science. The former will provide a priceless advantage in some analysis of harder but beam sensitive materials while the later will enable tomography of thicker specimen ( 1micron vs 200-300nm) as well as the possibility of identification of particulate if used in conjunction with EELS.

Case 1: STEM tomography of Golgi apparatus. This organelle is described as a stack of lamellae forming a structure of about 1-2 micron in diameter and involved in protein trafficking. The standard, even single section tomography gives a poor representation of the overall architecture and connectivity of this structure. Increase in expression level a Golgi associated protein, protein $\mathrm{X}$, change the morphology of the Golgi at optical microscopy level. STEM tomography reveals some insight in the new connectivity and the extent of the defects created by the increase of protein X expression (Figure 1) and underline the importance of this protein in overall protein maturation and trafficking

Case 2: Identification of fetuin-A containing calciprotein particle (CPP) in plastic sections. Accumulation of CPP is believed to drive inflammation and extra osseous mineral deposition. The activation of the inflammosome is triggered by internalization of the CPP, this study aim to understand the degradation pathway if the CCP. STEM-EELS was performed to identify the Ca and P components of the CPP and follow it during endocytosis. (Figure 2)

Case 3: Study of valence change in highly susceptible $\delta-\mathrm{MnO}_{2}: \delta-\mathrm{MnO}_{2}$ mineral, which is analogous to minerals produced by manganese oxidizing bacteria; is strongly oxidizing (containing Mn(IV)) and has been implicated in the redox transformation of a variety of organic and inorganic compounds in the environment. Oxidizing Mn(III/IV) minerals are also thought to be the primary environmental species involved in the release of toxic $\mathrm{Cr}(\mathrm{VI})$ by the oxidation of the less toxic and more insoluble $\mathrm{Cr}(\mathrm{III})$. We used a Gatan cryo Holder to maintain the sample temperature below -170 degres $\mathrm{C}$ in order to assess if cryo conditions were susceptible to stop the valence change due to beam damage. (Figure 3) 


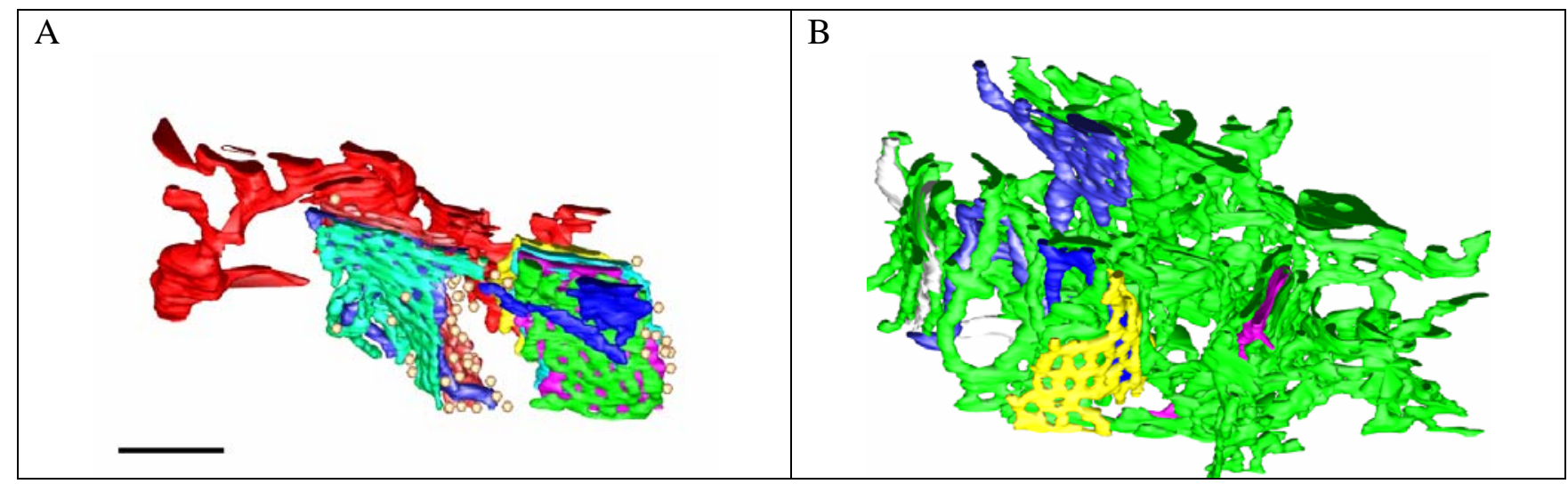

Figure 1. A) STEM tomography model from a 1 micron thick section showing a normal Golgi apparatus. Each color represent an independent cisternae. The ER is represented in red and is not directly linked to the Golgi. B) STEM tomography of protein X knock down. Each color represent an independent system. The extent of the green model shows a high level of interconnectivity between some cisternae of the Golgi apparatus and the ER. Scale bar: 500nm

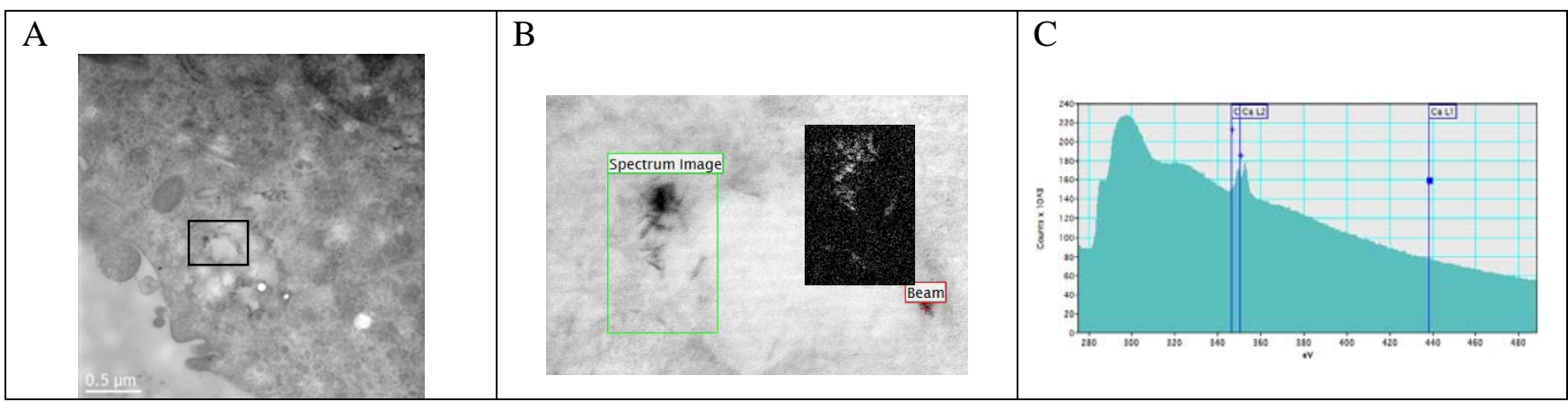

Figure 2 A) thin section of thru a monocyte in TEM bright field. The square represents the region acquire in STEM (B) and STEM-EELS (B inset) showing the Calcium signal. C) STEM-EELS spectra of an individual crystal.
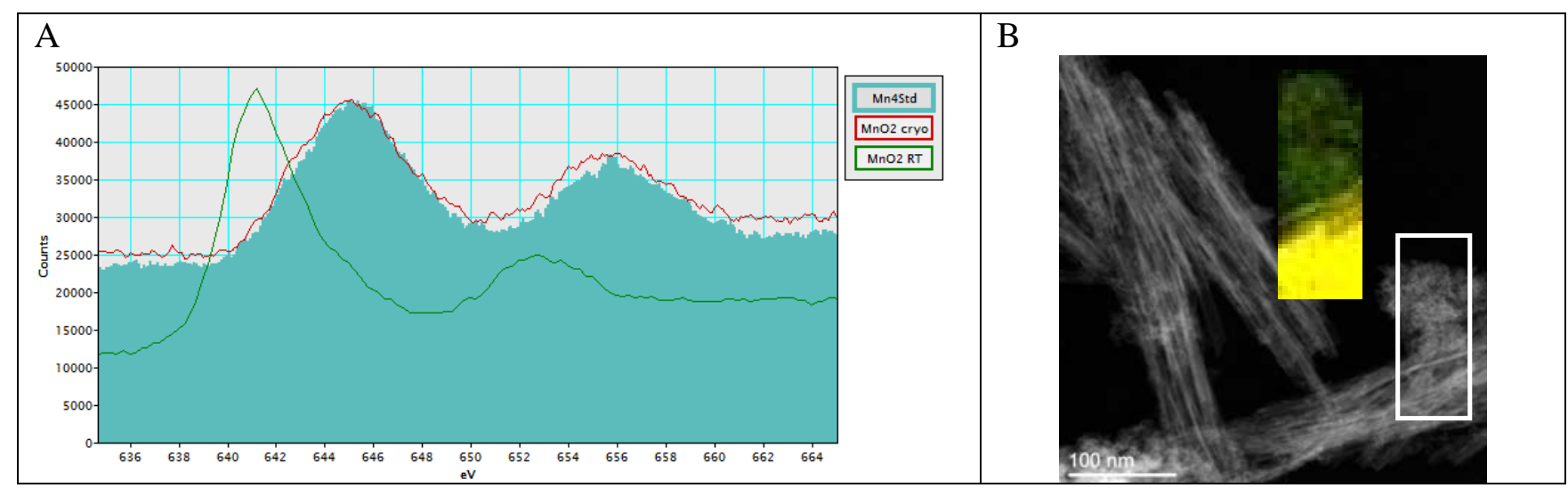

Figure 3. A) Manganese IV standard EELS spectrum (aqua) compared EELS spectra of $\delta$ - $\mathrm{MnO}_{2}$ maintained at room temperature or in cryo conditions. No valence change is observed in cryo conditions. B) Cryo STEM-EELS image of a sample containing both manganese II (red) and manganese IV (green) 